\title{
Flexible hysteroscopy with narrow band imaging (NBI) for endoscopic diagnosis of malignant endometrial lesions
}

\author{
IORI KISU $^{1}$, KOUJI BANNO ${ }^{1}$, YUSUKE KOBAYASHI ${ }^{1}$, ASUKA ONO $^{1}$, KENTA MASUDA $^{1}$, \\ ARISA UEKI $^{1}$, HIROYUKI NOMURA ${ }^{1}$, AKIRA HIRASAWA ${ }^{1}$, TAKAYUKI ABE ${ }^{2}$, \\ KEISUKE KOUYAMA ${ }^{2}$, NOBUYUKI SUSUMU ${ }^{1}$ and DAISUKE AOKI ${ }^{1}$ \\ ${ }^{1}$ Department of Obstetrics and Gynecology, ${ }^{2}$ The Center for Clinical Research, \\ School of Medicine, Keio University, Tokyo, Japan
}

Received October 14, 2010; Accepted December 3, 2010

DOI: $10.3892 /$ ijo.2011.903

\begin{abstract}
Narrow band imaging (NBI) for detection of blood vessels and microstructures on the mucosal surface is used in gastrointestinal endoscopy since it can improve qualitative diagnosis and detection of lesion. However, there are no studies on flexible hysteroscopy using NBI. We performed flexible hysteroscopy with NBI for outpatients to investigate the sensitivity and specificity of endoscopic diagnosis of malignant endometrial lesions. Of patients who attended our hospital for suspected lesions in the uterine cavity between April 2009 and May 2010, 104 subjects underwent hysteroscopy with NBI, in addition to white light. Using the pathological diagnosis as the gold-standard, we evaluated the sensitivity and specificity of NBI hysteroscopy for detecting atypical endometrial hyperplasia (AEH) or carcinoma. The results were also compared with historical data $(n=209)$ for conventional hysteroscopy using white light only in 2008. The sensitivities were $97.2 \%$ [95\% confidence interval (95\% CI): $90.3-99.7 \%$ ] and $82.6 \%$ (95\% CI: 74.4-89.0\%) for NBI hysteroscopy and conventional hysteroscopy, respectively. The $95 \%$ CIs for the two methods did not overlap and the sensitivity of lesion detection was higher with NBI hysteroscopy. Specificities were comparable, 90.6\% (95\% CI: $75.0-98.0 \%$ ) and $85.1 \%$ (95\% CI: 76.3-91.6\%) between the methods. NBI hysteroscopy has increased sensitivity for detection of atypical endometrial hyperplasia $(\mathrm{AEH})$ or carcinoma. A comparison with historical data suggested that NBI may be useful for diagnosis of malignant endometrial lesions. As far as we are aware, this is the first evaluation of flexible hysteroscopy with NBI for diagnosis of malignant endometrial lesions.
\end{abstract}

Correspondence to: Dr K. Banno, Department of Obstetrics and Gynecology, School of Medicine, Keio University, 35 Shinanomachi, Shinjuku-ku, Tokyo 160-8582, Japan

E-mail: kbanno@sc.itc.keio.ac.jp

Key words: narrow band imaging, hysteroscopy, endometrial lesions

\section{Introduction}

Hysteroscopy is an endoscopic examination of the uterine cavity using an endoscope for patients with suspected endometrial lesions and is used for patients with lesions detected by imaging, such as transvaginal ultrasonography, endometrial cytology or histology, and patients with hemorrhage, hypermenorrhea or infertility. Hysteroscopy has similar or better sensitivity than transvaginal ultrasonography, endometrial cytology or histology for detection of endometrial lesions (1-3). It is also useful for preoperative evaluation of lesions, identification of the occupation of tumors, or evaluation of the effect of high-dose medroxyprogesterone acetate (MPA) therapy. However, Lasmar et al have reported that the sensitivities of hysteroscopy for endometrial hyperplasia (EMH) and endometrial carcinoma are 56.3 and $80.0 \%$, respectively (4). This suggests that diagnostic criteria based on morphological changes in an endometrial lesion are inadequate and a pathological examination is required for diagnosis of endometrial lesions.

Narrow band imaging (NBI) is an endoscopic imaging technology produced by shifting the light spectrum to a narrow band. NBI allows enhanced imaging of blood microvessels and microstructures in the surface layer. In the gastrointestinal field, NBI has contributed to improving qualitative diagnosis and detection of lesions (5). The visible light wavelength for humans is $400-700 \mathrm{~nm}$, and different wavelengths appear as different colors. Light of $400 \mathrm{~nm}$ appears blue, that of $550 \mathrm{~nm}$ is green, and that of $600 \mathrm{~nm}$ is red, while light with a wide range of wavelengths of 400-700 $\mathrm{nm}$ appears white. In the NBI system, a filter inserted into the light path is positioned in front of a xenon lump, so that light of narrow bands at 415 and $540 \mathrm{~nm}$ reaches the mucosal surface. Oxidized hemoglobin in blood has an absorption spectrum with peaks at 415 and $540 \mathrm{~nm}$, and absorbs most of the blue light emitted in the NBI system, resulting in blood vessels appearing as a dark green color. Other tissue scatters the light and appears as a bright color. Therefore, NBI distinguishes blood vessels in the mucosal surface from other tissue.

In this study, NBI was used in flexible hysteroscopy in outpatients for enabling visual identification of blood vessels with clear contrast. The objective of this study was to evaluate 
the utility of NBI flexible hysteroscopy for endoscopic diagnosis of atypical endometrial hyperplasia (AEH) or endometrial carcinoma.

\section{Patients and methods}

Patients and procedures. The subjects were 104 patients with suspected endometrial lesions who were examined by NBI hysteroscopy in addition to conventional white light hysteroscopy from April 2009 to May 2010, and thereafter were pathologically diagnosed based on surgical specimens. The study was approved by the ethics committee of our hospital and was performed after obtaining the informed consent of the patients. Patients receiving high-dose MPA therapy and those with a history of this therapy were included in the study. Patients with a history of surgery including dilatation and curettage (D\&C) and transcervical resection of the endometrium (TCRE), and those for whom hysteroscopy was required, were also included. Patients with voluminous genital hemorrhage, menstruation, possible pregnancy, endometritis and cervical neoplasia were excluded from the study.

Pathological diagnoses were made using specimens obtained in surgery, including D\&C, TCRE, and hysterectomy. Based on these diagnoses, the cases were classified into four categories: benign lesion (normal endometrium, endometrial polyp, submucosal myoma, decidual change due to MPA therapy), EMH, AEH, and endometrial carcinoma. Endometrial lesions that did not fall into these categories were excluded. The sensitivity and specificity of hysteroscopy for diagnosis of AEH or endometrial carcinoma, for which high-dose MPA therapy or surgery are used, were examined for 104 subjects evaluated by NBI hysteroscopy from April 2009 to May 2010 and 209 subjects evaluated by white light hysteroscopy in 2008.

The instruments used in the study were a 3.1-mm flexible hysterofiberscope (HYF-XP) connected to a CCD digital camera (OTV-S7ProH-HD-12E), a VISERA Pro Xenon light source (CLV-S40Pro), and a VISERA Pro video processor (OTV-S7Pro) (all from Olympus Medical Systems Corp., Tokyo, Japan). An Olympus OEV-191H 19-in high-definition LCD monitor was used. Images were printed on an Olympus OEP-4 video printer and movies were recorded with a highdefinition recorder (AG-HMR10; Panasonic Co., Osaka, Japan) and a DVD recorder (DMR-XP12; Panasonic Co.).

Patients were placed in the lithotomy position and the examinations were performed transvaginally with no kind of anesthesia. Physiological saline solution was dripped from a bag suspended $1 \mathrm{~m}$ above the patient to have a constant intrauterine pressure, and the scope was inserted into the uterus while the uterine cavity was distended.

Diagnostic criteria. For diagnosis with hysteroscopy, we took notice of protruding lesions, atypical vessels and necrosis. The evaluation of blood vessels based on NBI hysteroscopy was used for diagnosis other than that of white light hysteroscopy. Malignant lesions frequently have atypical blood vessels with an irregular, expanded, stenosed, interrupted, or zigzagged shape, and judgment of these atypical vessels was made with reference to Fig. 1. We prepared the following diagnostic criteria (Fig. 2), based on our own experience and criteria published elsewhere (6): Normal endometrium: endometrial mucosa with a smooth surface and no evident vascular network. Endometrial polyp: protruding lesion with a smooth surface and no evident vascular network. Myoma: protruding lesion with a smooth surface and clearly evident vascular network consisting of vessels branching from main vessels, but no atypical vessels. Decidual changes due to MPA therapy: decidual membrane lesion with a smooth surface and evident vascular network with a basal layer in the cavity. EMH: thick proliferated endometrium with none or minor blood vessel network on the surface. AEH: thick endometrium or protruding lesion with evident atypical blood vessels on the surface, but no evident necrosis. Endometrial cancer: protruding lesion with a rough surface including evident atypical vessels and necrosis.

Statistical analysis. Descriptive statistics were calculated for demographic characteristics in each group (white light plus NBI and white light only hysteroscopy). The pathological diagnosis [4-scales: benign lesion (normal, polyp, myoma, decidual change), EMH, AEH, and carcinoma] was used to indicate the true disease status. To evaluate the agreement of the hysteroscopic diagnosis with the pathological data, crosstables were generated and weighted Kappa coefficients were estimated for each diagnostic test. Sensitivity and specificity were calculated with binary diagnostic data (disease defined as AEH or carcinoma). The $95 \%$ confidence intervals (CIs) for sensitivity, specificity and accuracy were estimated using the Clopper-Pearson (exact) method. All data were analyzed with SAS (version 9.1).

\section{Results}

Clinicopathological features of lesions. NBI hysteroscopy was performed in 104 subjects with no complications and observations were feasible in all cases. Cross tables of diagnostic results between each hysteroscopy and pathological diagnosis were summarized in Table I. Based on the pathological diagnoses, the subjects evaluated with NBI hysteroscopy included 25 benign lesions ( $24.0 \%$; normal endometrium 3, endometrial polyp 7, myoma 6, decidual change 9), and $7 \mathrm{EMH} \mathrm{(6.7 \% ),} 16$ AEH (15.4\%), and 56 carcinoma (53.8\%) subjects. The 209 subjects examined conventionally in 2008 included 87 benign lesions (41.6\%; normal endometrium 17, endometrial polyp 30, myoma 29, decidual change 11), and $7 \mathrm{EMH}$ (3.3\%), $32 \mathrm{AEH}$ $(15.3 \%)$, and 83 carcinoma (39.7\%) subjects.

Comparison of visualization by conventional and NBI hysteroscopy. Compared with the conventional method, NBI hysteroscopy visualized blood vessels in the endometrium surface as a dark green color with clear contrast, resulting in easier inspection of the blood vessels and detection of atypical vessels (Fig. 3).

Sensitivity, specificity and accuracy of NBI and conventional hysteroscopy. In the 104 subjects examined by NBI hysteroscopy from April 2009 to May 2010, the hysteroscopic and pathological diagnoses were concordant in 92 subjects (accuracy $88.5 \%, 95 \%$ CI: 82.3-94.6\%). For conventional hysteroscopy examined in 2008 , the diagnoses were concordant in 


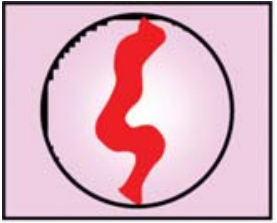

Expanded

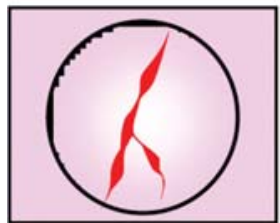

Stenosed

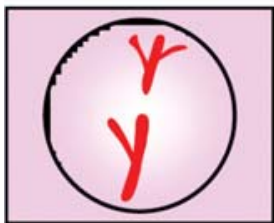

Interrupted

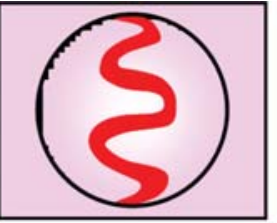

Tortuous

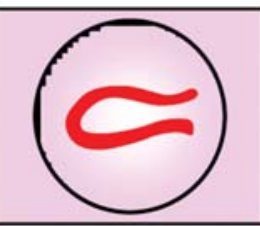

Inflectional

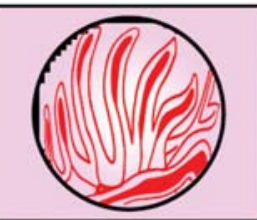

Papillary (sea anemone like)

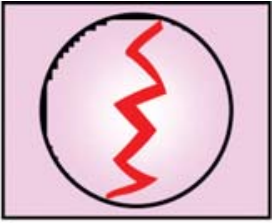

Zigzagged

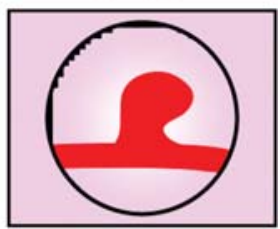

Varix like

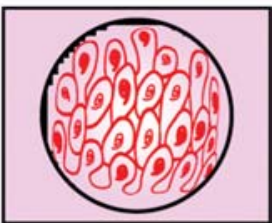

Coiling

(frog eggs like)
Figure 1. Atypical vessels. A variety of atypical blood vessels were observed in the mucosa of endometrial malignant lesions, including vessels with expanded, tortuous, and zigzagged shapes; variable diameter; inflectional, interrupted, papillary shapes; and sea anemone shapes.

148 of 209 subjects (accuracy 70.8\%, 95\% CI: 64.6-77.0\%). The weighted Kappa coefficients for the hysteroscopy and pathological diagnoses were 0.895 (95\% CI: 0.835-0.955) and 0.663 (95\% CI: 0.582-0.744) for NBI and conventional hysteroscopy, respectively. These results show that NBI hysteroscopy has better agreement with pathological diagnosis compared to the conventional hysteroscopy (Table I).

The sensitivities in detecting AEH or carcinoma for NBI and conventional hysteroscopy were $97.2 \%$ (95\% CI: 90.3$99.7 \%$ ) and $82.6 \%$ (95\% CI: 74.4-89.0\%), respectively, based
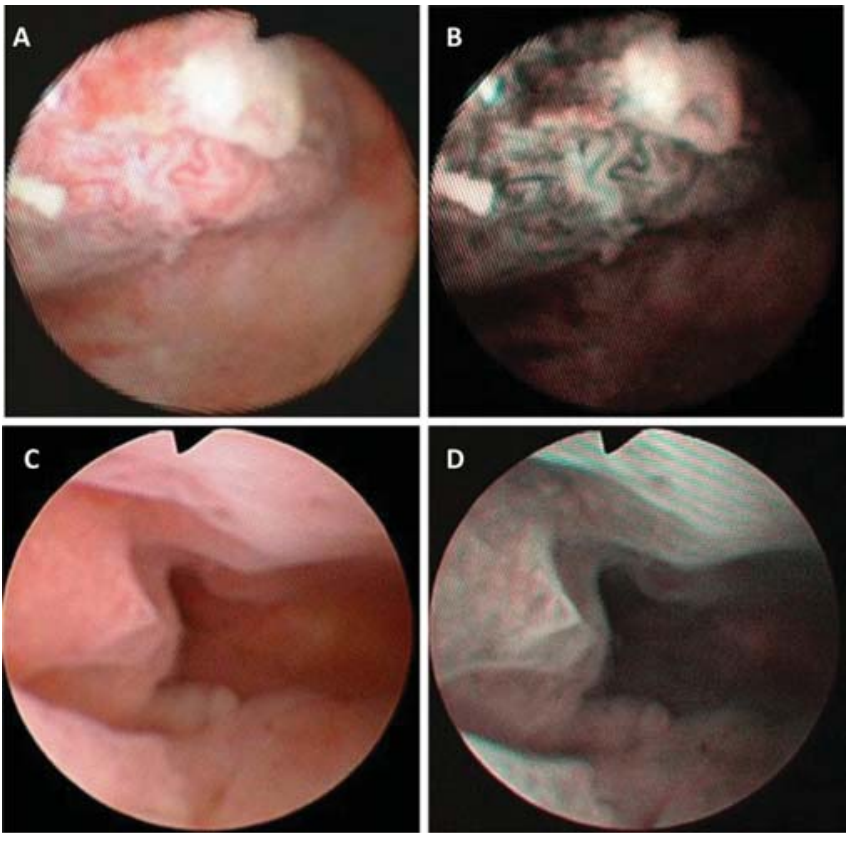

Figure 3. Comparison of visualization of endometrial lesions by conventional and NBI hysteroscopy. Endometrial carcinoma in conventional (A) and NBI (B) hysteroscopy. Evident necrosis and atypical blood vessels were found in the protruding lesion area. Atypical vessels were visualized more clearly by NBI. Endometrial hyperplasia in conventional (C) and NBI (D) hysteroscopy. Thick proliferated endometrial lesions were observed in the whole uterine cavity, but without observation of a prominent blood vessel network, even with NBI.

on pathological diagnosis of AEH or endometrial carcinoma, which are endometrial lesions that require therapy of high-dose MPA or surgery. NBI hysteroscopy had a higher sensitivity, and the lower limit of the $95 \%$ CI for NBI hysteroscopy was higher than the upper limit for the conventional method. The specificities of the two methods were similar: $90.6 \%$ (95\% CI: 75.0-98.0\%) and $85.1 \%$ (95\% CI: 76.3-91.6\%), respectively (Table II and Fig. 4).

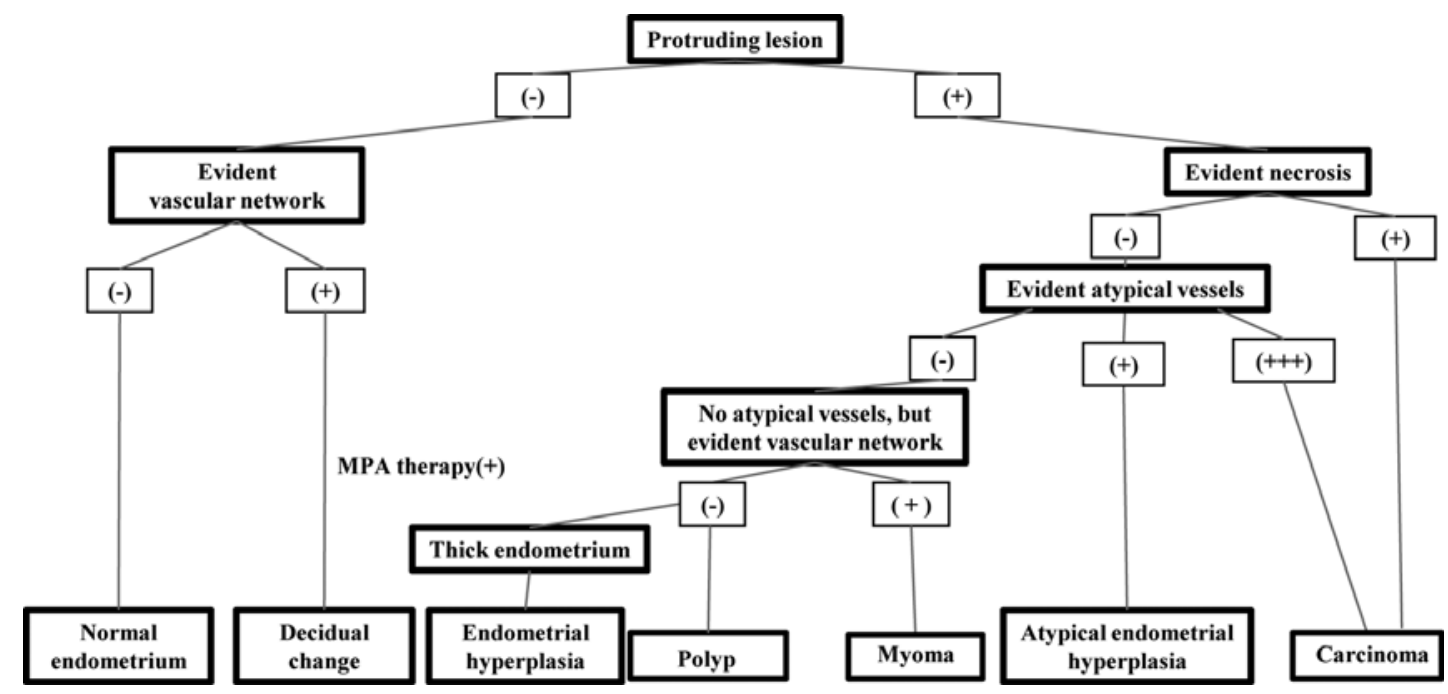

Figure 2. Diagnostic criteria for endometrial lesions detected by hysteroscopy. 
Table I. Comparison of conventional and NBI hysteroscopy diagnoses with pathological findings.

\begin{tabular}{cccccc}
\multicolumn{3}{c}{ Pathological diagnosis } & & \\
\hline Benign & EMH & AEH & Carcinoma & $\begin{array}{c}\% \text { Accuracy } \\
(95 \% \mathrm{CI})\end{array}$ & $\begin{array}{c}\text { Weighted Kappa } \\
(95 \% \mathrm{CI})\end{array}$
\end{tabular}

\begin{tabular}{|c|c|c|c|c|c|c|}
\hline \multicolumn{7}{|c|}{ Conventional hysteroscopy } \\
\hline Benign & 75 & 4 & 11 & 9 & & \\
\hline EMH & 0 & 1 & 0 & 0 & 70.8 & 0.663 \\
\hline $\mathrm{AEH}$ & 12 & 1 & 11 & 13 & $(64.6-77.0)$ & $(0.582-0.744)$ \\
\hline Carcinoma & 0 & 1 & 10 & 61 & & \\
\hline \multicolumn{7}{|c|}{ NBI hysteroscopy } \\
\hline Benign & 23 & 0 & 1 & 0 & & \\
\hline EMH & 1 & 5 & 1 & 0 & 88.5 & 0.8949 \\
\hline $\mathrm{AEH}$ & 1 & 2 & 12 & 4 & $(82.3-94.6)$ & $(0.835-0.955)$ \\
\hline Carcinoma & 0 & 0 & 2 & 52 & & \\
\hline
\end{tabular}

Table II. Sensitivity and specificity of conventional and NBI hysteroscopy for diagnosis of AEH or carcinoma.

\begin{tabular}{|c|c|}
\hline $\begin{array}{l}\text { Pathological } \\
\text { diagnosis }\end{array}$ & \\
\hline$\geq \mathrm{AEH}<\mathrm{AEH}$ & $\begin{array}{c}\% \text { Sensitivity } \% \text { Specificity } \\
(95 \% \mathrm{CI}) \quad(95 \% \mathrm{CI})\end{array}$ \\
\hline
\end{tabular}

Conventional

hysteroscopy

$\begin{array}{lllcc}\geq \text { AEH } & 95 & 14 & 82.6 & 85.1 \\ <\text { AEH } & 20 & 80 & (75.6-89.5) & (77.9-92.3)\end{array}$

NBI hystero-

scopy

\begin{tabular}{lrrcc}
$\geq$ AEH & 70 & 3 & 97.2 & 90.6 \\
$<$ AEH & 2 & 29 & $(90.3-99.7)$ & $(75.0-98.0)$ \\
\hline
\end{tabular}

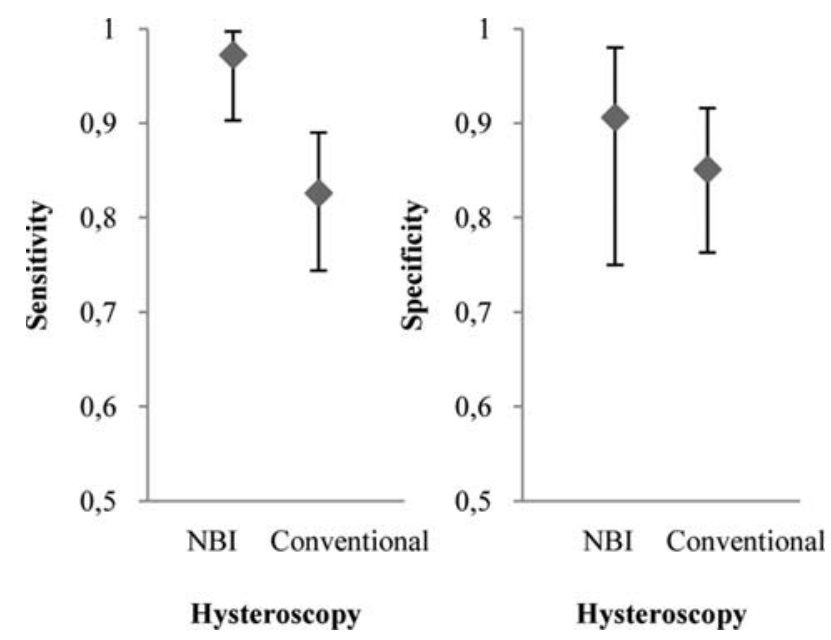

Figure 4. Sensitivity and specificity of NBI and conventional hysteroscopy for detecting atypical endometrial hyperplasia or carcinoma.

\section{Discussion}

This study provides a preliminary evaluation of NBI as imageenhanced endoscopic technique used in the gastrointestinal field for diagnosis of endometrial lesions. Development of NBI started in May 1999, and a NBI system was made available by Olympus Corp. in May 2006. This approach has been used effectively in the gastrointestinal field for endoscopic evaluation of the pharynx, esophagus, stomach, and large intestine, as shown in several studies. However, to our knowledge, only 7 reports (7-13) have described use of NBI in gynecology. This report is the first study to apply NBI flexible hysteroscopy to diagnosis of endometrial lesions in outpatients.

Improved visualization with NBI is achieved by shifting the light spectrum to narrow bands at 415 and $540 \mathrm{~nm}$, which results in clear discrimination of lesions, microstructures and blood microvessels. Most blue light emitted in the NBI system is absorbed by oxidized hemoglobin in blood, resulting in visualization of blood vessels as a dark green color, in contrast to the light scattered by other tissue in the mucosal membrane. Neoplastic vessels tend to proliferate on the surface of cancerous lesions in the digestive tract, and this makes NBI very useful for qualitative diagnosis of malignant and benign lesions through clear visualization of microvessels (14). In the gynecological field, angiogenesis is also considered as a diagnostic marker of a malignant endometrial lesion $(15,16)$, which suggests that NBI hysteroscopy may have a supplementary role in diagnosis of malignant endometrial lesions.

The choice of therapy for AEH and endometrial carcinoma is often high-dose progesterone and surgery including D\&C and hysterectomy. A malignant lesion should be suspected when proliferation of neoplastic atypical vessels is observed, and therefore identification of the atypical vessel using NBI is important for diagnosis and therapy. However, diagnosis with hysteroscopy tends to be subjective. For this reason, we defined diagnostic criteria for objective assessment based on classification of the atypical vessel (Figs. 1 and 2). 
The correct diagnostic rate were compared using data collected in two different periods. The sensitivity with NBI hysteroscopy of $88.5 \%$ (95\% CI: 82.3-94.6\%) was higher than that with the conventional hysteroscopy $70.8 \%$ (95\% CI: 64.6-77.0\%), and the lower limit of the confidence interval with NBI was higher than the upper limit with conventional hysteroscopy (Fig. 4). This result indicates that NBI hysteroscopy may improve the diagnostic accuracy compared with the conventional method. Furthermore, the weighted Kappa coefficient between NBI hysteroscopy and pathological diagnosis was as high as 0.8949 (95\% CI: 0.835-0.955), which indicates that the agreement of these diagnosis was high. The sensitivity and specificity of NBI hysteroscopy for detection of AEH or carcinoma were 97.2\% (95\% CI: 90.3-99.7\%) and 90.6\% (95\% CI:75.0-98.0\%), respectively, compared with $82.6 \%$ (95\% CI: $74.4-89.0 \%$ ) and $85.1 \%$ (95\% CI: 76.3$91.6 \%)$, respectively, for conventional hysteroscopy. Therefore, NBI hysteroscopy had higher sensitivity and similar specificity to those of the conventional method. This result suggests that NBI improves detection of malignant endometrial lesions, resulting from easier inspection of atypical vessels. The higher sensitivity but similar specificity compared to the conventional method suggests that NBI emphasizes vessel visualization, but does not increase the false positive rate. Overall, our results suggest that NBI hysteroscopy has superior sensitivity and accuracy compared with conventional hysteroscopy, and is useful for diagnosis of endometrial lesions.

Despite the promising results, our study has some limitations. This study involved only small number of patients and was conducted at a single institution. It was not performed as a randomized comparison, but as an exploratory comparison of NBI hysteroscopy with historical data obtained using conventional hysteroscopy. This design could lead to biases in the two groups based on the differences in time period, patients and operators, or sampling scheme. However, the sensitivity and specificity in the two groups are not influenced by sampling scheme, and data were collected from the two groups in the same hospital with an interval of only one year. It is unlikely that there are major biases in the data, but a crossover randomized study is required to confirm the results.

The high sensitivity of NBI hysteroscopy is likely to be clinically useful for judging whether an endometrial lesion is malignant or not, based on the following points. First, biopsy or endometrial histology may not be required for all patients with a suspected endometrial lesion, resulting in a decrease in unnecessary and invasive examinations. Second, judgment of the effect of therapy with high dose progesterone may be made by NBI hysteroscopy without the need for D\&C. Third, NBI hysteroscopy may improve the diagnostic accuracy and sensitivity of detection of endometrial lesions.

In the digestive tract, NBI as image-enhanced endoscopic technique has been used in magnifying endoscopy for evaluation of blood microvessels and surface microstructure, with the goals of qualitative diagnosis and evaluation of invasion depth. Magnifying NBI endoscopy is referred to as 'optical biopsy', and a classification based on magnifying NBI for each internal organ has been proposed (17-21). Improvements in endoscopic imaging technology also allow clear visualization of cells and nuclei using endocytoscopy and endomicroscopy $(17,22,23)$. These methods may allow evaluation as cytology and histology of biopsy specimens. We plan to investigate the diagnostic utility of the new imaging technology by applying NBI to magnifying hysteroscopy for evaluation of the microstructure and blood microvessels in endometrial lesions.

In conclusion, NBI hysteroscopy improved the diagnostic sensitivity for AEH or endometrial carcinoma, compared with conventional hysteroscopy. This increased sensitivity was achieved by application of NBI to enhance the contrast between vessels and other tissue in the lesion area, which resulted in easier detection of atypical vessels. Our results suggest that NBI hysteroscopy may be a useful supplementary endoscopic method for diagnosis of endometrial lesions. However, the study was performed as a historical comparative study of two groups, and large-scale, multicenter, prospective randomized controlled studies are required to establish the utility of NBI hysteroscopy for the diagnosis of endometrial lesions.

\section{Acknowledgments}

We thank Mr. Takashi Saga, Tomoharu Sanada and Kenji Matsushima of Olympus Medical Science Sales Co., Ltd. for technical assistance during the study. We also thank Drs Tai Ohmori and Tomohiko Nishi for advice on the NBI system. This study was supported by the Japan Society for the Promotion of Science (JSPS) through a Grant-in-Aid for Scientific Research (KAKENHI); a Grant-in-Aid for Scientific Research (C) (22591866); the Takeda Science Foundation; the Foundation for Promotion of Cancer Research; and the Japanese Foundation for Research and Promotion of Endoscopy (JFE) Grant.

\section{References}

1. Clark TJ, Voit D, Gupta JK, Hyde C, Song F and Khan KS: Accuracy of hysteroscopy in the diagnosis of endometrial cancer and hyperplasia: a systematic quantitative review. JAMA 288: 1610-1621, 2002.

2. Garuti G, Sambruni I, Colonnelli M and Luerti M: Accuracy of hysteroscopy in predicting histopathology of endometrium in 1500 women. J Am Assoc Gynecol Laparosc 8: 207-213, 2001.

3. Van Donqen H, De Kroon CD, Jacobi CE, Trimbos JB and Jansen FW: Diagnostic hysteroscopy in abnormal uterine bleeding: a systematic review and meta-analysis. BJOG 114: 664-675, 2007.

4. Lasmar RB, Barrozo PR, De Oliveira MA, Coutinho ES and Dias R: Validation of hysteroscopic view in cases of endometrial hyperplasia and cancer in patients with abnormal uterine bleeding. J Minim Invasive Gynecol 13: 409-412, 2006.

5. Gono K, Obi T, Yamaguchi M, et al: Appearance of enhanced tissue features in narrow-band endoscopic imaging. J Biomed Opt 9: 568-577, 2004.

6. Dotto JE, Lema B, Dotto JE Jr and Hamou J: Classification of microhysteroscopic images and their correlation with histologic diagnoses. J Am Assoc Gynecol Laparosc 10: 233-246, 2003.

7. Barrueto FF and Audlin KM: The use of narrowband imaging for identification of endometriosis. J Minim Invasive Gynecol 15: 636-639, 2008.

8. Surico D, Viqone A and Leo L: Narrow band imaging in endometrial lesions. J Minim Invasive Gynecol 16: 9-10, 2009.

9. Cicinelli E, Tinelli R, Colafiqlio G, Pastore A, Mastrolia S, Lepera A and Clevin L: Reliability of narrow-band imaging (NBI) hysteroscopy: a comparative study. Fertil Steril (In press).

10. Kuroda K, Kitade M, Kikuchi I, et al: Vascular density of peritoneal endometriosis using narrow-band imaging system and vascular analysis software. J Minim Invasive Gynecol 16: 618-621, 2009. 
11. Kuroda K, Kitade M, Kikuchi I, Kumakiri J, Matsuoka S, Kuroda $\mathrm{M}$ and Takeda S: Peritoneal vascular density assessment using narrow-band imaging and vascular analysis software, and cytokine analysis in women with and without endometriosis. J Minim Invasive Gynecol 17: 21-25, 2010.

12. Farrugia M, Nair M and Kotronis KV: Narrow band imaging in endometriosis. J Minim Invasive Gynecol 14: 393-394, 2007.

13. Surico D, Vigone A, Bonvini D, Tinelli R, Leo L and Surico N Narrow-band imaging in diagnosis of endometrial cancer and hyperplasia: a new option? J Minim Invasive Gynecol (In press).

14. Muto M, Katada C, Sano Y and Yoshida S: Narrow band imaging: a new diagnostic approach to visualize angiogenesis in superficial neoplasia. Clin Gastroenterol Hepatol 3: 16-20, 2005.

15. Abukafia O, Triest WE and Sherer DM: Angiogenesis in malignancies of the female genital tract. Gynecol Oncol 72: 220-231, 1999

16. Stefansson IM, Salvesen HB and Akslen LA: Vascular proliferation is important for clinical progress of endometrial cancer. Cancer Res 66: 3303-3309, 2006.

17. ASGE Technology Committee, Kwon RS, Wong Kee Song LM, et al: Endocytoscopy. Gastrointest Endosc 70: 610-613, 2009.
18. Inoue H, Kudo SE and Shiokawa A: Technology insight: laserscanning confocal microscopy and endocytoscopy for cellular observation of the gastrointestinal tract. Nat Clin Pract Gastroenterol Hepatol 2: 31-37, 2005.

19. Muto M, Nakane M, Katada C, et al: Squamous cell carcinoma in situ at oropharyngeal and hypopharyngeal mucosal sites. Cancer 101: 1375-1381, 2004.

20. Inoue $\mathrm{H}$, Honda $\mathrm{T}$, Nagai $\mathrm{K}$, et al: Ultra-high magnification endoscopic observation of carcinoma in situ of the esophagus. Dig Endosc 9: 16-18, 1997.

21. Yao K, Anagnostopoulos GK and Tagunath K: Magnifying endoscopy for diagnosing and delineating early gastric cancer. Endoscopy 41: 462-467, 2009.

22. Sano Y, Horimatsu T, Fu KI, et al: Magnifying observation of microvascular architecture of colorectal lesions using a narrow band imaging system. Dig Endosc 18: S44-S51, 2006.

23. Inoue H, Sasajima K, Kaga M, et al: Endoscopic in vivo evaluation of tissue atypia in the esophagus using a newly designed integrated endocytoscope: a pilot trial. Endoscopy 38: 891-895, 2006 